\title{
OVERVIEW OF THE SYNERGIA 3-D MULTI-PARTICLE DYNAMICS MODELING FRAMEWORK*
}

\author{
P. Spentzouris ${ }^{\dagger}$, J. Amundson, FNAL, Batavia, IL 60510, USA \\ D. Dechow, Tech-X, Boulder, CO 80303, USA
}

\begin{abstract}
High precision modeling of space-charge effects is essential for designing future accelerators as well as optimizing the performance of existing machines. Synergia is a high-fidelity parallel beam dynamics simulation package with fully three dimensional space-charge capabilities and a higher-order optics implementation. We describe the Synergia framework and model benchmarks we obtained by comparing to semi-analytic results and other codes. We also present Synergia simulations of the Fermilab Booster accelerator and comparisons with experiment.
\end{abstract}

\section{THE SYNERGIA FRAMEWORK}

Synergia [1] is a framework for state-of-the-art simulation of linear and circular accelerators with a fully threedimensional (3D) treatment of space charge, and the capability to use arbitrary order maps for the single-particle optics modeling. It is designed to be a general-purpose tool with an interface that is accessible to accelerator physicists who are not experts in simulation and computing techniques. Space-charge calculations are computationally intensive, typically requiring the use of parallel computers. The implementation of Synergia utilizes Particle-In-Cell (PIC) techniques and is fully parallel, including the particle tracking and space-charge modules. The code itself is a hybrid system based on the IMPACT [2] space-charge code and the mxyzptlk/beamline libraries [3], which includes a MAD parser. Synergia includes enhancements to these codes as well as new modules. The space-charge module uses the path length along the reference trajectory, $s$, as the independent variable and implements a variety of different boundary conditions. Synergia has multi-turn injection capabilities and can follow multiple bunches longitudinally.

The user-level interface to Synergia consists of a set of Python classes that wrap the low-level interfaces to the various packages used. The Python interface generates an input file that is read by the simulation itself. The Python interpreter need not be present at run time. The Python interface can even generate a job to be automatically transferred and submitted to a remote machine where no Python interpreter is available. Synergia also includes a build system that allows it to be compiled and run on various platforms without requiring the user to modify the code and/or build system.

\footnotetext{
${ }^{*}$ Work supported in part by the US DOE Office of High Energy Physics, under the auspices of the Scientific Discovery through Advanced Computing Program

† spentz@fnal.gov
}

\section{Performance}

For high precision 3D simulations, large numbers of macroparticles, on the order of $10^{6-7}$, and fine spacecharge grids, typical size of $33 \times 33 \times 257$, are required. In order to obtain the necessary computing power for such simulations, we have ported our code to different parallel machines, including commodity PC clusters, as well as specialized parallel computers. A summary of the performance of the Synergia code for FNAL Booster modeling is shown in Fig. 1, as a function of the number of processors used to run the simulation. The performance depends on

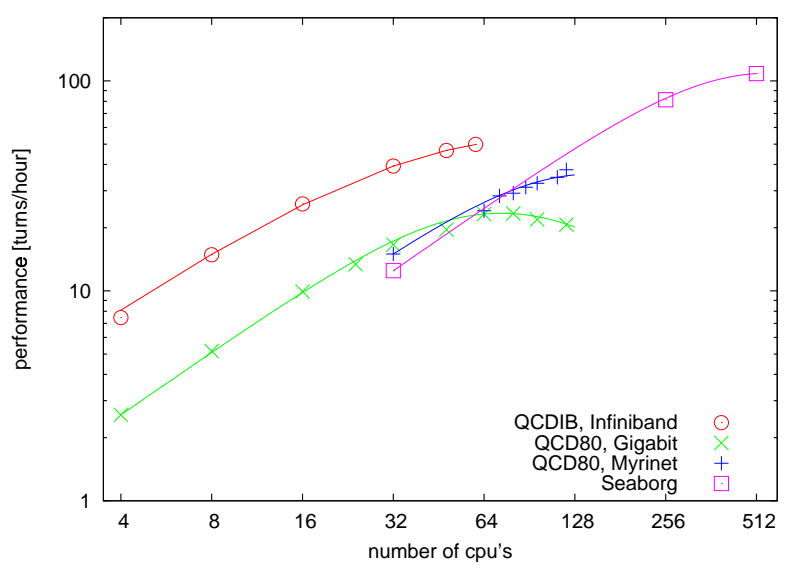

Figure 1: Synergia performance, measured in Booster turns per hour, versus number of processors, for different parallel machines.

an interplay of networking speed, latency, and processing speed. For our Booster simulations, peak performance of $\sim 100$ Booster turns per hour is obtained running on 512 processors on the NERSC supercomputer.

\section{Benchmarking}

In order to verify the accuracy of our simulation we provide a test suite, in which we model several cases simple enough to perform comparisons with semi-analytic calculations [1]. In all these comparisons, the Synergia code is in good agreement with the theoretical expectations. An example of such a comparison is shown in Fig. 2. Here we model a cold, uniform density, $100 \mathrm{~mA}$ proton beam, with kinetic energy of $250 \mathrm{MeV}$, in a FODO channel with rf cavities, and compare with the MaryLie/IMPACT (ML/I) [4] 3D code; the agreement is excellent. The cavity phases have been set so that the first cavity accelerates the beam and the second decelerates it by the same amount. Since 


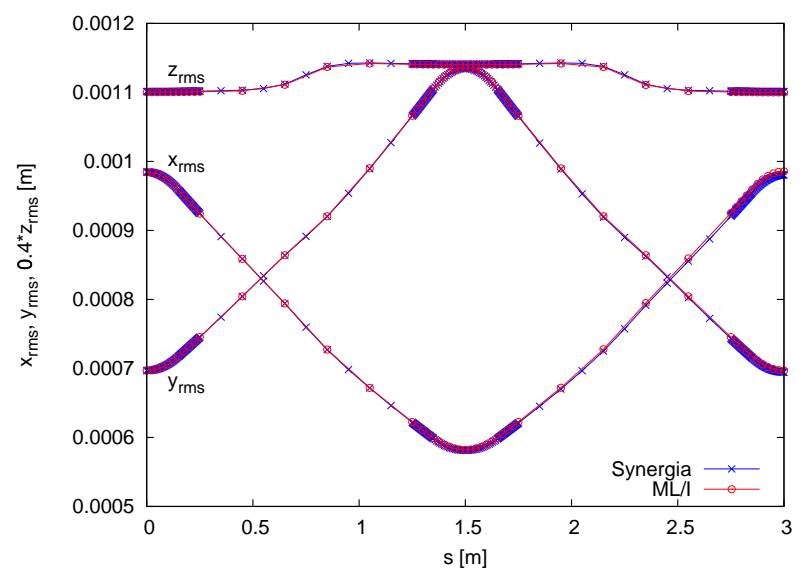

Figure 2: Comparison of the Synergia and MaryLie/IMPACT predictions for the r.m.s. beam envelopes of a cold beam propagating in a FODO channel with rf cavities.

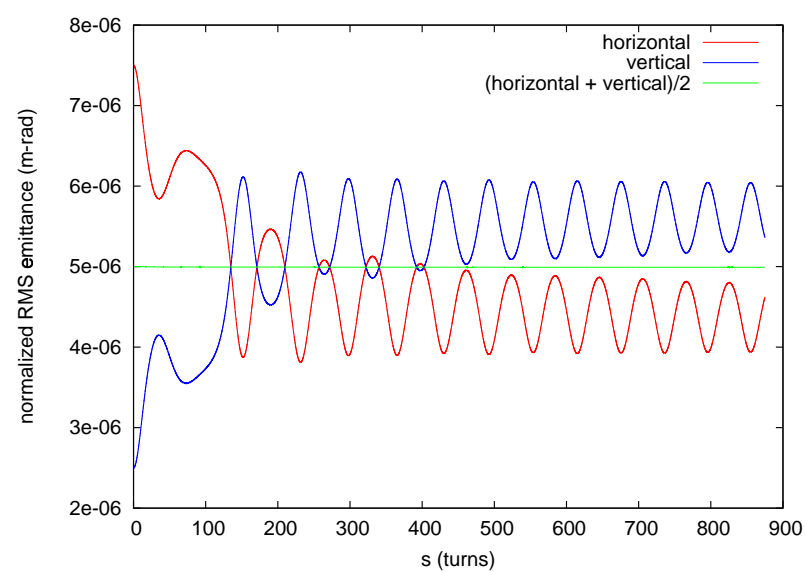

Figure 3: Normalized horizontal and vertical 2-D emittances and their average, versus turn number, showing the emittance exchange due to the Montague resonance.

the beam is cold and uniform, the rms equations describe the problem exactly, so there is a matched condition where the final envelopes are identical to the initial values. We used a 3D envelope matching code to find the matched beam parameters. We generated a numerical realization of the matched uniform distribution consisting of 100,000 particles. These particles were used as the input of both Synergia and ML/I. We have also compared Synergia predictions of the space-charge driven Montague resonance at the CERN PS with other major simulation codes [5]. In Fig. 3 we show the normalized vertical and horizontal normalized emittances and their average, versus turn, for a Synergia CERN PS simulation using a linear lattice with $Q_{x}=6.207$ and $Q_{y}=6.21$, one of the cases used in the comparison.

\section{FERMILAB BOOSTER SIMULATIONS}

The Booster [6] is a rapid-cycling, $15 \mathrm{~Hz}$, alternating gradient synchrotron with a radius of 75.47 meters. The lattice consists of 96 combined function magnets in 24 periods, with nominal horizontal and vertical tunes of 6.9 and 6.7 respectively. The Booster accelerates protons from a kinetic energy of $400 \mathrm{MeV}$ to $8 \mathrm{GeV}$, using $17 \mathrm{rf}$ cavities with frequency that slews from $37.8 \mathrm{MHz}$ at injection to $52.8 \mathrm{MHz}$ at extraction. Typically, the injection process lasts for ten Booster turns, resulting to a total average current of $420 \mathrm{~mA}$. The injected beam is a stream of bunches equally spaced at the linac RF frequency of 201.2 MHz.

In this section we study how space-charge affects the Booster beam during the first 500 turns of the cycle (injection, capture, and bunching). For the simulations we use an idealized Booster lattice without any non-linear elements, but we do employ second order maps and we use a beam with realistic energy spread.

\section{Emittance dilution}

First we investigate how space-charge affects the emittance of the Booster without including rf. In Fig. 4 we plot the normalized 4-D transverse emittance ${ }^{1}$ for five different initial beam conditions, described in the caption of the figure. As expected, in the cases where the beam was matched there is no emittance growth. (Our matching procedure takes into account space-charge effects on the second moments of the beam). In the mismatched cases, with a $20 \%$ mismatch, we observe a $12 \%$ increase of the beam emittance during the first 10 to 15 turns after injection. The effect is a combination of chromatic and space-charge effects and it is very similar for both the single- and multiturn injection cases. The total current is the same, 0.420 Amps, in both cases. The emittance growth can be related to the conversion of beam free energy from mismatch oscillations into thermal energy of the beam, due to the effect of the non-linear space-charge forces [7]. We compare our result with the prediction of the free-energy model for the breathing mode case. With a mismatch parameter of 1.2, as in the case of our simulation, the model predicts a 4D transverse emittance growth of $13 \%$, in good agreement with the Synergia result.

Including $\mathrm{rf}$ in the simulation increases the space-charge effects (bunching) and introduces a stronger coupling between the horizontal (bending) and the longitudinal planes. The rf is turned on 20 turns after injection, which lasts for 10 turns, and capture lasts for 200 turns. In the capture process cavity pairs start paraphased and are brought linearly in phase. In Fig. 5 we show the evolution of a longitudinal plane slice, $2 \pi$ wide in the $37.8 \mathrm{Mhz}$ rf phase. During the debunching period, we see the bunches of the injected linac beam become "s-shaped" due to space charge. After the beam is captured in the $37.8 \mathrm{Mhz}$ rf, it also develops

\footnotetext{
${ }^{1}$ Defined as the square root of the determinant of the covariance matrix of the transverse phase space.
} 


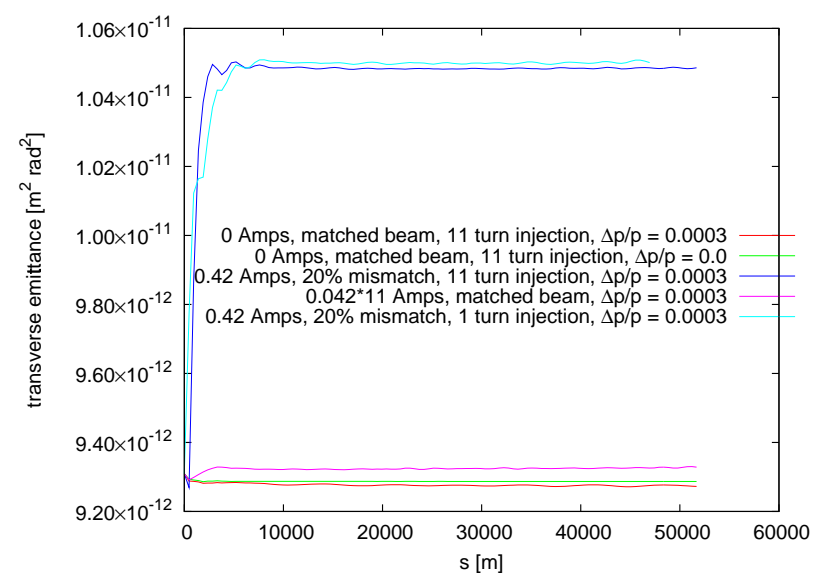

Figure 4: Normalized 4-D transverse emittance in $\mathrm{m}^{2} \mathrm{rad}^{2}$ for different initial conditions. The red and green curves correspond to a matched beam, with space-charge effects turned off, with and without a momentum spread of 0.0003 , respectively. The purple and light blue curves correspond to a beam of 0.420 Amps total current and momentum spread of 0.0003 , matched and mismatched respectively. Multi-turn injection of 11 turns is used in all of the above cases. The dark blue curve corresponds to a single turn injection simulation of a 0.420 Amp mismatched beam with 0.0003 momentum spread.
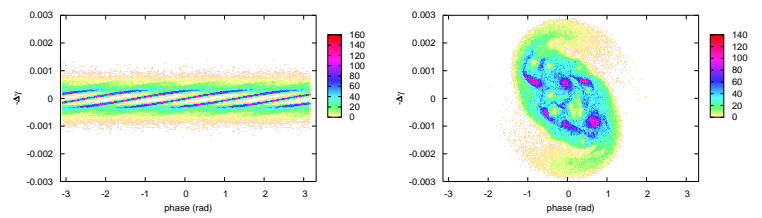

Figure 5: Longitudinal phase-space after 18 turns (left) and after 385 turns (right).

"s-shape" characteristics, but maintains some structure due to the "folding" of the linac bunches. The effects of the rf on the normalized 4-D transverse emittance is shown in Fig. 6; the emittance growth is $\sim 2.5$ times larger $(30 \%$ versus $12 \%$ ) than in the case with no rf. In addition, as also shown in Fig. 6, the emittance varies, following the variation of the longitudinal beam width, due to the correlation between longitudinal and horizontal phase-space planes. The variation starts with a period of 20 turns, and ends with a period of 5 turns.

Finally, we compare the simulated horizontal beam width with a measurement using the Booster Ionization Profile Monitor, Fig. 7. The agreement is very good. A discussion of the experimental technique is presented in [8].

\section{REFERENCES}

[1] J. Amundson, P. Spentzouris, J. Qiang, R. Ryne, FNAL-Pub-04/136-CD, submit-

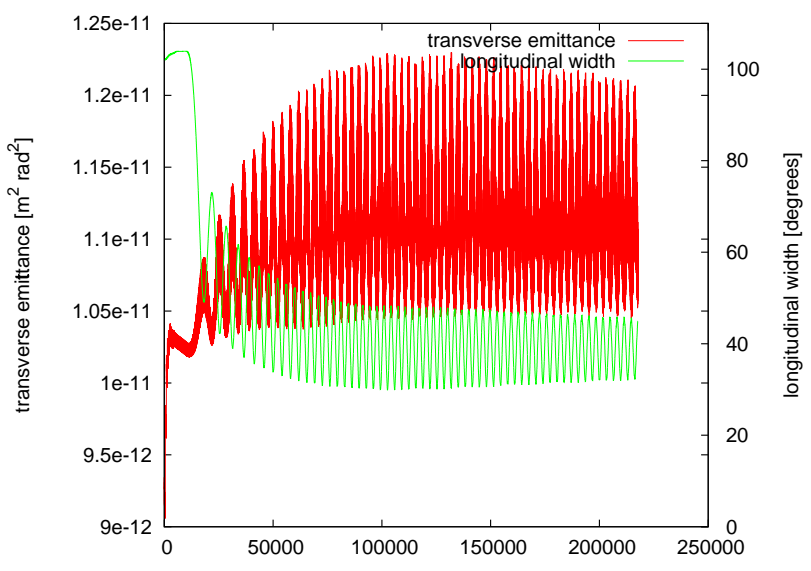

Figure 6: Normalized 4-D transverse emittance in $\mathrm{m}^{2} \mathrm{rad}^{2}$ and longitudinal beam width in degrees versus $\mathrm{s}$, for a Booster simulation including rf.

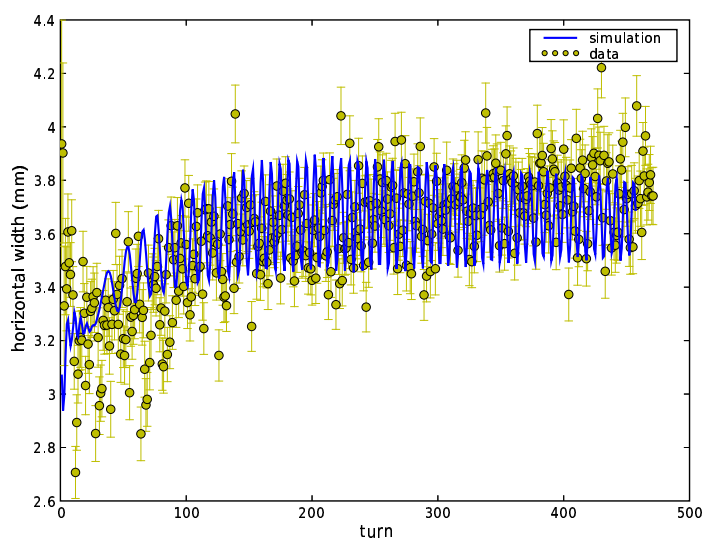

Figure 7: Booster horizontal beam width in $\mathrm{mm}$ versus turn number compared to the Synergia prediction.

ted to the Journal of Computational Physics; http://cepa.fnal.gov/psm/aas/Advanced_Accelerator_Simulation.html

[2] J. Qiang, R. D. Ryne, S. Habib and V. Decyk, J. Comp. Phys. 163, 434 (2000).

[3] L. Michelotti, FERMILAB-CONF-91-159.

[4] http://scidac.nersc.gov/accelerator/mli/manual.pdf

[5] Ingo Hofmann, et al, "Benchmarking of Simulation Codes Based on the Montague Resonance in the CERN-PS", these proceedings.

[6] Booster Staff 1973 Booster Synchrotron ed E L Hubbard Fermi National Accelerator Laboratory Technical Memo TM405

[7] M. Reiser, "Theory and Design of Charged Particle Beams" Weiley, N.Y. 1994, pp. 470-473; M. Reiser J. Appl. Phys. 70 (1991) 1919.

[8] J. Amundson and P. Spentzouris, "Space Charge Experiments and Simulation in the Fermilab Booster", these proceedings. 